Cite as: Stephane H Maes, (2020), “Gravity Dictates the Number of Fermion Generations: 3”, viXra:2007.0068v1, https://vixra.org/pdf/2007.0068v1.pdf or https://shmaesphysics.wordpress.com/2020/06/24/gravity-dictates-thenumber-of-fermion-generations-3/, June 24, 2020.

\title{
Gravity Dictates the Number of Fermion Generations: 3
}

\author{
Stephane H. Maes ${ }^{1}$
}

June 24, 2020

\begin{abstract}
:
In a multi-fold universe, gravity emerges from Entanglement through the multi-fold mechanisms. As a result, gravity-like effects appear in between entangled particles that they be real or virtual. Long range, massless gravity results from entanglement of massless virtual particles. Entanglement of massive virtual particles leads to massive gravity contributions at very smalls scales. Multi-folds mechanisms also result into a spacetime that is discrete, with a random walk fractal structure and non-commutative geometry that is Lorentz invariant and where spacetime nodes and particles can be modeled with microscopic black holes. All these recover General relativity at large scales and semi-classical model remain valid till smaller scale than usually expected. Gravity can therefore be added to the Standard Model. This can contribute to resolving several open issues with the Standard Model.

It has always been intriguing to explain why there seems to be only 3 generations of Fermions, for each family, including neutrinos. In this paper, we show that there are only 3 regimes defined in the Standard Model Lagrangian complemented with gravity, when it comes to the contribution of fermion masses interacting with Higgs bosons. As a result, differentiations of mass implies only 3 generations. It is another surprising result, from adding nonnegligible gravity to the Standard model. While shown in the context of a multi-fold universe, the result can be extended to any model where gravity is not negligible at small scales.
\end{abstract}

\section{Introduction}

The new preprint [1] proposes contributions to several open problems in physics like the reconciliation of General Relativity (GR) with Quantum Physics, explaining the origin of gravity proposed as emerging from quantum (EPREinstein Podolsky Rosen) entanglement between particles, detailing contributions to dark matter and dark energy and explaining other Standard Model mysteries without requiring New Physics beyond the Standard Model other than the addition of gravity to the Standard Model Lagrangian. All this is achieved in s multi-fold universe that may well model our real universe, which remains to be validated.

With the proposed model of [1], spacetime and Physics are modeled from Planck scales to quantum and macroscopic scales and semi classical approaches appear valid till very small scales. In [1], it is argued that spacetime is discrete, with a random walk-based fractal structure, fractional and noncommutative at, and above Planck scales (with a 2-D behavior and Lorentz invariance preserved by random walks till the early moments of the universe). Spacetime results from past random walks of particles. Spacetime locations and particles can be modeled as microscopic black holes (Schwarzschild for photons and spacetime coordinates, and metrics between Reisner Nordstrom [2] and Kerr Newman [3] for massive and possibly charged particles - the latter being possibly extremal). Although surprising, [1] recovers results consistent with others like [4], while also being able to justify the initial assumptions of black holes from the gravity or entanglement model. The resulting gravity model recovers General Relativity at larger scale, as a 4-D process, with massless gravity, but also with massive gravity

\footnotetext{
${ }^{1}$ shmaes.physics@gmail.com
} 
components at very small scale that make gravity significant these scales. Semi-classical models also work well till way smaller scales that usually expected.

In this paper, we remain at a high level of discussion of the analysis and references are generic for the subjects. It makes the points accessible to a wider audience and keeps the door open to further papers or discussions devoted to details of interest. Yet, it requires the reader to review [1], as we do not revisit here all the details of the multifold mechanism or reconstruction of spacetime. More targeted references for all the material discussed here are compiled in [1].

\section{2. $\mathrm{SM}_{\mathrm{G}}$ : The Standard Model with Multi-Fold Gravity}

[1] proposes that in a multi-fold universe, the Lagrangian is complemented by terms associated to gravity and entanglement (in the form of the sum of the attractive effective potentials) [1].

$$
\mathscr{L}_{\text {total }}=\mathscr{L}_{S M}+\mathscr{L}_{\text {Gravity-with-MF }}+\mathscr{L}_{\text {EPR }}+\mathscr{L}_{\text {Others... }}
$$

The effect of gravity can be seen through the attractive potential contributions of all the energy sources. It can also been seen as expressing the Standard Model Lagrangian in curved spacetime (semi-classical point of view), now considered valid till small scales.

EPR entanglement is not believed to often play a significant role, except in dark matter use cases [5].

The last term is all other "New Physics" terms and we will consider it to be null.

With (1), the mass generation terms now involves the gravity effective potential described in [1]:

$$
\begin{aligned}
\mathscr{L}_{\text {int }} & \propto C_{1}(\text { vertex }) \\
& +m_{\text {acquired }}\left(1+\frac{V_{\text {eff }}}{m_{\text {acquired }}}\right)\left(C_{2}(\text { lepton } / \text { quark })\right. \\
& +C C)
\end{aligned}
$$

Where, the $C_{1}$ () designates the vertex contribution that represents the interaction with the Higgs/QCD Vacuum and/or Quark Gluon condensate with chirality flip and $C_{2}$ () represents the contributions of the right-handed + lefthanded leptons or quarks.

\section{The Number of Fermion Generations}

This work is inspired from the recent publication from Steven Weinberg trying unsuccessfully to model and estimate Leptons and Quark masses [6].

We considered the success encountered so far by adding gravity to the standard model as already reported in [1] (e.g. stabilization of the Electroweak vacuum), to explain how neutrinos acquire mass without New Physics [7], Dark matter [5] and Energy [8], without New Physics, and the absence of proton decays [9] or magnetic monopoles [10]; along with careful analyses that reduce plausibility of New Physics through (Grand Unification Theories) GUTS [11], (Theories of Everything) ToEs as well as superstring theories [12]. It seemed tempting to see if $\mathrm{SM}_{\mathrm{G}}$ has better luck than [6]. 
So we start from equation (2) and write it as a function of the fermion mass and the Higgs mass interacting to provide mass to the (charged) fermions. Note that with [7], we argue that this statement extends to neutrinos.

It can be expressed as:

$$
\mathscr{L}_{\text {int }} \propto m_{\text {Fermion }}+V_{\text {eff }}(\text { gravity })+V_{\text {eff }}(E P R)
$$

Or,

$$
\mathscr{L}_{\text {int }} \propto m_{\text {Fermion }}\left(1+M_{\text {Higgs }} K_{1}+K_{2}\right)
$$

Where, $M_{\text {Higgs }}$ designates the Higgs Mass. $K_{1}$ and $K_{2}$ depend on the interaction including the mass of fermion.

(4) can be estimated and plotted with the following considerations: For $m_{\text {Fermion }} \approx M_{\text {Higgs }}$, the potentials effect is strong and maximum (as the particles have equivalent masses and will overlap a lot when the fermion bumps into a Higss boson). For $m_{\text {Fermion }} \approx 0$, the effect is essentially null. In between, the effect is essentially not impacted by $m_{\text {Fermion }}$ (dominated by Higgs attraction, no charge of overlap when mass changes). The result is plotted in in Figure 1.

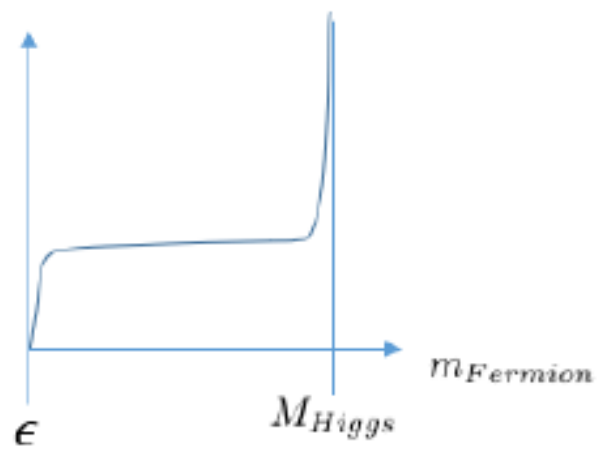

Figure 1: Estimated plot of equation (4) as a function of $m_{\text {Fermion. }}$.

As a result, we can see that only 3 regimes exist and hence differentiated fermion generations can only distribute in 3 regions.

The existence of 3 and only 3 generations of Fermions per family is automatically derived from Figure 1. It is achieved within the context of the Standard Model with Gravity, in a multi-fold universe, and without the need of New Physics.

Also, this analysis is for a Multi-fold universe as in [1]. [1] details arguments and ways to check its relationship with the real universe. Besides properties that can be experimentally verified (in the future because of the macroscopic weakness of gravity and gravity like effects for entangled systems), [1] shows how the multi-fold mechanisms and behaviors are in many aspects in today's conventional physics, that, at times, anticipates the behaviors modeled of a multi-fold universe. In addition, [1] explains many results obtained in gravity, quantum mechanics, General Relativity, superstring theory, Loop Quantum Gravity and the AdS/CFT correspondence conjecture. All these works attempt to come up with models for the real universe. It is at least a good sign that [1] may provide an interesting model of the real universe. 
Other theories showing that gravity is relevant at the level of the standard model, can repeat the arguments, even with no relation to multi-fold universe and its mechanisms or to gravity emergence from entanglement. So our model here is generic: if we add gravity to Standard Model with a model keeping it non negligible at the Standard Model scales, then there will be 3 and only 3 generations of Fermions.

If our model here is not validated by experience, it would not invalidate the multi-fold mechanisms and the proposal that gravity emerges from entanglement as detailed in [1]. The analysis builds on [1], as a consequence of it, but it is not a condition for validation of multi-fold universes.

\section{Conclusions}

We believe that [1] makes a compelling case for the consistency of its multi-fold proposal. The present paper shows how the mechanisms of multi-fold universes can predict the existence of 3 and only 3 generations of Fermions (including neutrinos per [7]).

The model works for multi-fold universe as well as in any situation where gravity is non negligible and added to the Standard Model.

This along with similar results in [1] and [5,7-11], make a strong case for more seriously considering the implications of adding gravity to the Standard Model to obtain $\mathrm{SM}_{\mathrm{G}}$, as a way to contribute to addressing open issues and offer better alternatives to New Physics speculations. This goes hand in hand with recognizing that this also implies the need to seriously consider that gravity may not always be negligible at the Standard Model scales as proposed in [1].

References: (most references come from popular science to make the discussion more approachable) [1]: Stephane H. Maes, (2020) “Quantum Gravity Emergence from Entanglement in a Multi-Fold Universe", viXra:2006.0088v1, (June 9, 2020). Updates and revisions tracked at https://shmaesphysics.wordpress.com/2020/06/09/paper-published-as-preprint-quantum-gravity-emergencefrom-entanglement-in-a-multi-fold-universe/

[2]: https://en.wikipedia.org/wiki/Reissner\%E2\%80\%93Nordstr\%C3\%B6m metric

[3]: https://en.wikipedia.org/wiki/Kerr-Newman_metric

[4]: Burinskii, Alexander, (2008), "The Dirac-Kerr-Newman electron", arXiv:0507109v4

[5]: Stephane H Maes, (2020), "Explaining Dark Matter Without New Physics?", viXra:2007.0006v1, https://shmaesphysics.wordpress.com/2020/06/19/explaining-dark-energy-small-cosmological-constant-andinflation-without-new-physics/, June 21, 2020.

[6]: Steven Weinberg, (2020), "Models of Lepton and Quark Masses", arXiv:2001.06582v1

[7]: Stephane H Maes, (2020), "Right-handed neutrinos? Mass? Ask Gravity", viXra:2007.0018v1, https://shmaesphysics.wordpress.com/2020/06/21/right-handed-neutrinos-ask-gravity/, June 21, 2020.

[8]: Stephane H Maes, (2020), "Explaining Dark Energy, Small Cosmological Constant and Inflation Without New Physics?", viXra:2006.0261, https://shmaesphysics.wordpress.com/2020/06/19/explaining-dark-energy-smallcosmological-constant-and-inflation-without-new-physics/, June 19, 2020.

[9]: Stephane H Maes, (2020), "Gravity Induced Anomalies Smearing in Standard Model so that Protons May Never Decay, Except in Black Holes ", viXra:2006.0128v1, https://shmaesphysics.wordpress.com/2020/06/12/protonsmay-never-decay-except-in-black-holes/, June 13, 2020.

[10]: Stephane H Maes, (2020), "Gravity or Magnetic Monopoles? You Cannot Have Both!“, viXra:2006.0190v1, https://shmaesphysics.wordpress.com/2020/06/15/gravity-or-magnetic-monopolesyou-cannot-have-both/, June 15, 2020. 
[11]: Stephane H Maes, (2020), "Ultimate Unification: Gravity-led Democracy vs. UberSymmetries", viXra:2006.0211v1, https://shmaesphysics.wordpress.com/2020/06/16/ultimate-unification-gravityled-democracy-vs-uber-symmetries/, June 16, 2020.

[12]: Stephane H Maes, (2020), “Dualities or Analogies between Superstrings and Multi-fold

Universe", viXra:2006.0178v1, https://shmaesphysics.wordpress.com/2020/06/14/dualities-or-analogies-betweensuperstrings-and-multi-fold-universes/, June 14, 2020. 